\title{
OPEN Association of cardiac injury with hypertension in hospitalized patients with COVID-19 in China
}

Xiaofang Zeng ${ }^{1,2}$, Anandharajan Rathinasabapathy ${ }^{3}{ }^{3}$, Dongliang Liu ${ }^{4}$, Lihuang Zha ${ }^{1,2}$, Xiangwei Liu ${ }^{1}$, Yiyang Tang ${ }^{1,2}$, Famei $\mathrm{Li}^{1,2}$, Wenchao $\mathrm{Lin}^{1,2}$, Zaixin $\mathrm{Yu}^{1,2}$ \& Huiling Chen ${ }^{5 凶}$

Outbreak of global pandemic Coronavirus disease 2019 (COVID-19) has so far caused countless morbidity and mortality. However, a detailed report on the impact of COVID-19 on hypertension (HTN) and ensuing cardiac injury is unknown. Herein, we have evaluated the association between HTN and cardiac injury in 388 COVID-19 $(47.5 \pm 15.2$ years) including 75 HTN and 313 normotension. Demographic data, cardiac injury markers, other laboratory findings, and comorbidity details were collected and analyzed. Compared to patients without HTN, hypertensive-COVID-19 patients were older, exhibited higher C-reactive protein (CRP), erythrocyte sedimentation rate, and comorbidities such as diabetes, coronary heart disease, cerebrovascular disease and chronic kidney disease. Further, these hypertensive-COVID-19 patients presented more severe disease with longer hospitalization time, and a concomitant higher rate of bilateral pneumonia, electrolyte disorder, hypoproteinemia and acute respiratory distress syndrome. In addition, cardiac injury markers such as creatine kinase (CK), myoglobin, lactic dehydrogenase (LDH), and $\mathrm{N}$-terminal pro brain natriuretic peptide were significantly increased in these patients. Correlation analysis revealed that systolic blood pressure correlated significantly with the levels of CK, and LDH. Further, HTN was associated with increased LDH and CK-MB in COVID- 19 after adjusting essential variables. We also noticed that patients with elevated either high sensitivity-CRP or CRP demonstrated a significant high level of LDH along with a moderate increase in CK $(p=0.07)$ and CK-MB $(p=0.09)$. Our investigation suggested that hypertensive patients presented higher risk of cardiac injury and severe disease phenotype in COVID-19, effectively control blood pressure in HTN patients might improve the prognosis of COVID-19 patients.

The outbreak of novel coronavirus disease 2019 (COVID-19), set by severe acute respiratory syndrome coronavirus type 2 (SARS-CoV-2) has transformed into a global pandemic since it emerged in Wuhan, China in December 2019 ${ }^{1}$. Accruing data from the Center for Systems Science and Engineering at Johns Hopkins University has reported over 200 million confirmed COVID-19 cases and 4 million deaths global wide, till date (https://coronavirus.jhu.edu/map.html). SARS-CoV-2 is much more contagious than SARS-CoV and Middle East Respiratory Syndrome Coronavirus because of its mutation in the receptor binding domain and the subsequent acquisition of furin cleavage site in the $S$ spike protein ${ }^{2,3}$. Although the vaccine is available, the emergence of SARS-CoV-2 variants, such as SARS-CoV-2 spike protein substitution D614G, are still a threat for the global health and economy $y^{4,5}$.

Recent investigation demonstrates that cardiovascular comorbidities increase the susceptibility and severity of COVID-19 and also associated with poor disease prognosis ${ }^{6}$. It is well understood that both SARS-CoV and SARS-CoV-2 use angiotensin-converting enzyme 2 (ACE2) as a receptor to gain entry into the host system? Physiologically, ACE2 acts as a potent vasodilator converting Angiotensin II (Ang II) to Angiotensin 1-7 and hence, negatively regulates renin-angiotensin-aldosterone (RAAS) and renin-angiotensin system (RAS) system ${ }^{8}$. Ang II is a potent vasoconstricting octapeptide hormone that plays a pivotal role in the pathogenesis of hypertension $(\mathrm{HTN})^{9,10}$. HTN has been recently identified as the most frequent comorbidity in individuals with COVID$19^{11,12}$ and it increases the odds ratio for death by $3.05^{13}$. Further, patients with pre-existing HTN represent a

\footnotetext{
${ }^{1}$ Department of Cardiology, Xiangya Hospital, Central South University, Changsha, China. ${ }^{2}$ National Clinical Research Center for Geriatric Disorders, Xiangya Hospital, Central South University, Changsha, China. ${ }^{3}$ Division of Allergy, Pulmonary, and Critical Care Medicine, Vanderbilt University Medical Center, Nashville, TN, USA. 'Department of Spine Surgery, Xiangya Hospital, Central South University, Changsha, China. ${ }^{5}$ Department of Geriatric, Xiangya Hospital, Central South University, 87 Xiangya Road, Changsha 410008, Hunan, China. ${ }^{\boxplus}$ email: huilingchen@csu.edu.cn
} 
large proportion of patients who incur severe COVID-19 disease symptoms and disproportionately experience worse outcome than one without $\mathrm{HTN}^{13-15}$.

Cardiac injury occurs at an early stage of COVID-19, such that rate of incidence noticed during admission at $16.44 \%$ rose up to $25.53 \%$ with those non-survivors ${ }^{16}$. Similarly, another study with a big cohort of 416 patients reported a 19.7\% incidence of cardiac injury in COVID-19 patients. These reports suggest that concurrent myocardial damage could be an independent risk factor in hospital mortality ${ }^{12}$. Although a precise underlying mechanism driving cardiac injury in COVID-19 remains obscure, plausible reasons could be viral infection or a preexisting cardiovascular disease or the combination of both and this is worsened in the patients, who have an exaggerated cytokine storm ${ }^{4}$. However, two recent independent postmortem examinations demonstrated the absence of viral particles in cardiac tissues irrespective of a pronounced local tissue inflammation ${ }^{17,18}$ which suggests that deteriorating pre-existing cardiovascular disease could be the primary cause of cardiac injury in COVID-19 patients. Since ACE2 is extensively expressed in the heart tissue and plays a role in cardiovascular homeostasis, we decided to investigate the role of HTN in SARS-CoV-2 mediated myocardial damage in COVID19 patients. As determined, this study examined in depth on the potential association between HTN and cardiac injury in patients with COVID-19.

\section{Methods}

Study design and participants. In this multicenter retrospective study, 388 laboratory-confirmed COVID-19 patients admitted in the designated hospitals between January $16^{\text {th }}$ and March $14^{\text {th }}, 2020$ were randomly enrolled. Patients, who were 18 years or older were included in this investigation. The diagnosis for COVID-19 was strictly followed in accordance with World Health Organization interim guidance ${ }^{19}$ and the principles outlined in the Declaration of Helsinki. This study protocol was approved by the Ethics Committee of Xiangya Hospital, Central South University, Hunan and the informed written consent was obtained from all study participants.

Data collection. The medical records of 388 COVID-19 patients were retrospectively reviewed and analysed. Epidemiological (age and sex) as well as clinical information (symptoms, chronic medical histories, laboratory findings and other complications) along with the radiological results, documented during hospitalization were collected and verified independently by two physicians. Based on the seventh edition of the Chinese National Health Commission (https://www.scirp.org/reference/referencespapers.aspx?referenceid=2851516), patients were categorized into severe disease status, if they meet any of the following criteria: 1) shortness of breath, respiratory rate $\geq 30$ beats per $\min ; 2$ ) oxygen saturation $\leq 93 \%$ at rest; 3 ) arterial oxygen partial pressure/ oxygen concentration $\leq 300 \mathrm{mmHg}(1 \mathrm{mmHg}=0.133 \mathrm{kPa}) ; 4)$ lung images showing obvious progress of lesion size $>50 \%$ within $24-48 \mathrm{~h}$. and patients were classified into mild disease status, if they presented mild clinical symptoms or mild/no lesions on imaging findings.

Cardiac injury biomarker data such as creatine kinase (CK), creatine kinase-MB (CK-MB), myoglobin, lactate dehydrogenase (LDH), and N-terminal pro brain natriuretic peptide (NT-proBNP) were assessed immediately after hospitalization. Cardiac troponin I (cTnI) was not accounted in this study, since most of the patients presented within the normal range of $\mathrm{cTnI}$ and that was indicated as $<0.01 \mathrm{ng} / \mathrm{mL}$. Criteria of acute respiratory distress syndrome (ARDS) was followed as mentioned in the Berlin definition ${ }^{18}$. All patients were carefully reviewed for their chronic medical history (coronary heart disease, arrhythmia, hyperlipidaemia, HTN diabetes, chronic obstructive pulmonary disease, chronic kidney disease, chronic liver disease, and cerebrovascular disease) and divided into two groups based on their pre-existing HTN. All clinical features and study outcomes were compared between hypertensive- and normotensive-COVID-19 patients.

Subgroup analysis on the correlation between inflammation and cardiac injury was conducted in COVID19 patients concurrent with HTN. Specifically, hypertensive COVID-19 patients were divided into two groups based on their elevated level of either C-reactive protein (CRP) or high sensitivity-CRP (hs-CRP), and cardiac injury markers were compared.

Statistical analysis. Statistical analyses were performed using SPSS version 20.0 statistical software (SPSS Inc., Chicago, IL, USA). Descriptive statistics was obtained for all study variables. Continuous variables were presented as mean \pm standard deviation (SD), if they were normally distributed; otherwise, they were presented as median \pm range interquartile (IQR), and categorical variables as frequencies. All categorical variables between the patients with and without HTN were analyzed using Fisher exact test or $\chi 2$ test and continuous variables were compared using t-test or Mann-Whitney U test, as appropriate. The Spearman correlation coefficient was calculated to disclose relationship of inflammatory or cardiac injury markers with blood pressure. Further, binary logistic regression model was performed to determine the association between blood pressure and cardiac injury in these patients after adjusting essential variables. Statistical charts were generated using Prism 7 (GraphPad Software Inc., San Diego, CA, USA). A two-sided $p<0.05$ was considered statistically significant for the entire data set.

\section{Results}

Patients characteristics. Our investigation presented a total of 388 hospitalized COVID-19 patients (197 male and 191 female) with an average age, $47.5 \pm 15.2$ years. Fever and cough were the most common symptoms observed in $227(58.4 \%)$ and $217(55.8 \%)$ patients, followed by fatigue, sputum production and shortness of breath $67(17.2 \%), 56(14.4 \%)$ and $36(9.3 \%)$, respectively. Symptoms such as muscle ache, diarrhea, chest tightness, sore throat, rhinorrhea and headache were rare and noted in $16(4.1 \%), 10(2.6 \%), 7(1.8 \%), 15(3.9 \%), 10$ $(2.6 \%)$ and $16(4.1 \%)$ patients, respectively. We also noticed several other comorbidities such as diabetes, chronic 


\begin{tabular}{|c|c|c|c|c|}
\hline Variables & Total $(\mathrm{n}=388)$ & Normotension $(n=313)$ & Hypertension $(n=75)$ & $p$ value \\
\hline Age (mean, year) & $47.5 \pm 15.2$ & $44.5 \pm 11.9$ & $60.2 \pm 14.2$ & $<0.001$ \\
\hline Gender (male), n (\%) & $197(50.8 \%)$ & $159(50.8)$ & $38(50.7)$ & 0.984 \\
\hline SBP (mean, mmHg) & $126.6 \pm 14.8$ & $123.8 \pm 13.2$ & $138.5 \pm 15.2$ & $<0.001$ \\
\hline DBP ( mean, mmHg) & $79.7 \pm 10.9$ & $78.2 \pm 10.2$ & $86.2 \pm 11.4$ & $<0.001$ \\
\hline Temperature (mean, ${ }^{\circ} \mathrm{C}$ ) & $37.0 \pm 0.69$ & $37.0 \pm 0.7$ & $36.9 \pm 0.8$ & 0.498 \\
\hline Heart rate (mean, bpm) & $86.7 \pm 12.7$ & $86.0 \pm 12.1$ & $89.8 \pm 14.9$ & $\leq 0.05$ \\
\hline \multicolumn{5}{|l|}{ Symptoms (n, \%) } \\
\hline Fever & $227(58.4)$ & $126(40.3)$ & $40(53.3)$ & $\leq 0.05$ \\
\hline Cough & $217(55.8)$ & $173(55.3)$ & $44(58.7)$ & 0.361 \\
\hline Fatigue & $67(17.2)$ & $256(81.8)$ & $10(13.3)$ & $<0.001$ \\
\hline Sputum production & $56(14.4)$ & $7(2.2)$ & $9(12.0)$ & $<0.001$ \\
\hline Shortness of breath & $36(9.3)$ & $4(1.3)$ & $13(17.3)$ & $<0.001$ \\
\hline Muscle ache & $16(4.1)$ & $13(4.2)$ & $3(4.0)$ & 0.952 \\
\hline Diarrhea & $10(2.6)$ & $8(2.6)$ & $2(2.7)$ & 0.957 \\
\hline Chest tightness & $7(1.8)$ & $4(1.3)$ & $3(4.0)$ & 0.135 \\
\hline Sore throat & $15(3.9)$ & $14(4.5)$ & $1(1.3)$ & 0.321 \\
\hline Rhinorrhea & $10(2.6)$ & $7(2.2)$ & $1(1.3)$ & 0.621 \\
\hline Headache & $16(4.1)$ & $14(4.5)$ & $2(2.7)$ & 0.747 \\
\hline \multicolumn{5}{|l|}{ Any comorbidity (n, \%) } \\
\hline Diabetes & $50(12.9)$ & $27(8.6)$ & $23(30.7)$ & $<0.001$ \\
\hline Chronic liver disease & $27(6.9)$ & $23(7.3)$ & $4(5.3)$ & 0.800 \\
\hline Coronary heart disease & $22(5.7)$ & $6(1.9)$ & $16(21.3)$ & $<0.001$ \\
\hline Chronic obstructive pulmonary disease & $22(5.7)$ & $15(4.8)$ & $7(9.3)$ & 0.160 \\
\hline Hyperlipidaemia & $13(3.3)$ & $11(3.5)$ & $2(2.7)$ & 0.957 \\
\hline Cerebrovascular disease & $13(3.3)$ & $6(1.9)$ & $7(9.3)$ & $<0.005$ \\
\hline Chronic kidney disease & $10(2.6)$ & $5(1.6)$ & $5(6.7)$ & $\leq 0.05$ \\
\hline Arrhythmia & $3(0.8)$ & $2(0.6)$ & $1(1.3)$ & 0.476 \\
\hline
\end{tabular}

Table 1. Baseline characteristics of patients infected with COVID-19.

liver disease, coronary heart disease, chronic obstructive pulmonary disease, hyperlipidemia, cerebrovascular disease, chronic kidney disease and arrhythmia in at least 50 (12.9\%), 27 (6.9\%), 22 (5.7\%), 22 (5.7\%), 13 (3.3\%), $13(3.3 \%), 10(2.6 \%)$ and $3(0.8 \%)$ patients, respectively. Rest of the baseline characteristics are outlined in the Table 1.

Although the mean systolic and diastolic blood pressure and heart rate of entire study subjects were in the normal range (systolic blood pressure, $126.6 \pm 14.8 \mathrm{mmHg}$, diastolic blood pressure, $79.7 \pm 10.9 \mathrm{mmHg}$ and heart rate, $86.7 \pm 12.7$ beats per minute), a detailed analysis revealed that 75 (19.3\%) patients were hypertensives (Table 1). A systemic comparison of normotensive COVID-19 patients with the hypertensives demonstrated that later were older $(44.5 \pm 11.9 v s .60 .2 \pm 14.2, p<0.001)$ and presented higher heart rate $(86.0 \pm 12.1 v s .89 .8 \pm 14.9$, $p<0.05)$, incidence of fever $(40.3 \% v s .53 .3 \%, p<0.05)$, sputum production $(2.2 \% v s .12 .0 \%, p<0.001)$ and shortness of breath $(1.3 \%$ vs. $17.3 \%, p<0.001)$. In addition, increased frequency was noticed for comorbidity such as diabetes ( 8.6 vs. $30.7 \%, p<0.001)$, coronary heart disease $(1.9$ vs. $21.3 \%, p<0.001)$, cerebrovascular disease ( 1.9 vs. $9.3 \%, p<0.001)$ and chronic kidney disease $(1.6$ vs. $6.7 \%, p<0.05)$ in hypertensive-COVID-19 patients. Surprisingly, neither the mean temperature of whole study subjects nor the normotensive $v s$. hypertensive groups were different from one other (Table 1).

Laboratory and radiographic findings. We next sought to investigate the metrics of laboratory and radiography assessments. As expected, hypertensive-COVID-19 patients demonstrated a significant higher level of CRP ( 12.45[5.00-38.60] vs. 9.30[2.31-23.68], $p<0.05)$, hs-CRP (12.34[4.20-37.13] vs. 5.38[0.50-22.48], $p<0.05)$, erythrocyte sedimentation rate $(46.00[25.25,70.00] v s .38 .00[18.00-60.00], p<0.05)$ and alanine aminotransferase (26.65[16.00-42.25] vs. 24.00[16.00-34.00], $p<0.05)$ (Table 2). Other biochemical and blood factors such as creatinine, uric acid, urea nitrogen, white blood cells and neutrophils demonstrated an increasing trend in the hypertensives, although those were statistically insignificant. Rest of the laboratory metrics such as lymphocytes, platelets, erythrocytes, hemoglobin, electrolyte ions, lipid molecules and etc., demonstrated a subtle or no change (Table 2). Further, radiography assessments demonstrated an increased prevalence of bilateral pneumonia $(85.6$ vs. $97.3 \%, p<0.001)$ in patients with HTN.

Complications and clinical outcome. Although the presence of HTN subtly altered the meantime from onset of symptom to hospitalization $(5.3 \pm 3.6 v s$. $5.8 \pm 3.8$ days, $p=0.399)$, it significantly increased mean hospitalization time $(15.9 \pm 7.1$ vs. $18.7 \pm 8.0$, days, $p<0.001)$. Further, hypertensive-COVID-19 patients displayed a severe disease status $(12.1$ vs. $76.0 \%, p<0.001)$ and presented other complications such as electrolyte disorder 


\begin{tabular}{|c|c|c|c|c|}
\hline Variables, median (IQR) & Total $(n=388)$ & Normotension $(n=313)$ & Hypertension $(n=75)$ & $p$ value \\
\hline White blood cells & $4.77(3.70-6.26)$ & $4.65(3.65-6.26)$ & $5.05(3.89-6.35)$ & 0.443 \\
\hline Neutrophil & $3.14(2.32-4.47)$ & $3.10(2.26-4.41)$ & $3.44(2.40-4.59)$ & 0.624 \\
\hline Lymphocyte & $1.07(0.77-1.46)$ & $1.06(0.78-1.48)$ & $1.10(0.74-1.34)$ & 0.446 \\
\hline Platelets & $178.00(142.00-225.00)$ & $178.00(142.99-225.50)$ & $178.00(135.00-223.00)$ & 0.509 \\
\hline Haemoglobin & $132.50(121.00-143.00)$ & $133.00(122.00-144.00)$ & $129.00(121.00-139.00)$ & 0.708 \\
\hline CRP & $9.65(2.98-26.70)$ & $9.30(2.31-23.68)$ & $12.45(5.00-38.60)$ & $\leq 0.05$ \\
\hline Hs-CRP & $6.00(0.61-23.75)$ & $5.38(0.50-22.48)$ & $12.34(4.20-37.13)$ & 0.03 \\
\hline $\mathrm{K}^{+}$ & $3.96(3.62-4.25)$ & $3.95(3.63-4.25)$ & $3.98(3.59-4.28)$ & 0.762 \\
\hline $\mathrm{Na}^{+}$ & $138.80(136.50-140.60)$ & $138.70(136.50-140.40)$ & $138.85(136.30-140.85)$ & 0.554 \\
\hline $\mathrm{Cl}^{-}$ & $103.15(100.00-105.30)$ & $103.40(100.23-105.40)$ & $101.85(99.10-104.65)$ & 0.195 \\
\hline $\mathrm{Ca}^{+}$ & $2.22(2.11-2.36)$ & $2.23(2.11-2.34)$ & $2.22(2.10-2.42)$ & 0.178 \\
\hline ESR $(\mathrm{mm} / \mathrm{h})$ & $40.00(20.00-64.00)$ & $38.00(18.00-60.00)$ & $46.00(25.25,70.00)$ & $\leq 0.05$ \\
\hline Total protein, $(\mathrm{g} / \mathrm{L})$ & $69.50(64.80-74.10)$ & $74.40(65.10-74.65)$ & $66.25(63.68-71.70)$ & 0.381 \\
\hline Albumin, $\mathrm{g} / \mathrm{L}$ & $40.50(37.12-44.40)$ & $40.80(37.90-44.63)$ & $38.95(34.45-42.05)$ & 0.382 \\
\hline Activated partial thromboplastin time, s & $31.80(27.80-36.43)$ & $32.05(28.03-36.85)$ & $30.30(26.80-35.35)$ & 0.325 \\
\hline $\mathrm{D}$-dimer, mg/L & $0.35(0.20-0.59)$ & $0.33(0.18-0.59)$ & $0.42(0.27-0.67)$ & 0.381 \\
\hline Fibrinogen $(\mathrm{g} / \mathrm{L})$ & $3.69(2.86-4.71)$ & $3.67(2.75-4.72)$ & $4.00(3.10-4.66)$ & 0.461 \\
\hline Alanine aminotransferase, U/L & $24.00(19.00-34.00)$ & $24.00(19.00-32.00)$ & $27.50(20.8-43.50)$ & $\leq 0.05$ \\
\hline Aspartate aminotransferase, $\mathrm{U} / \mathrm{L}$ & $24.00(16.00-35.00)$ & $24.00(16.00-34.00)$ & $26.65(16.00-42.25)$ & 0.184 \\
\hline Total bilirubin, mmol/L & $10.81(7.90-17.60)$ & $11.15(7.75-17.30)$ & $10.60(8.24-18.46)$ & 0.747 \\
\hline Creatinine, $\mu \mathrm{mol} / \mathrm{L}$ & $64.44(54.5-77.90)$ & $63.00(53.78-75.20)$ & $72.00(57.25-86.45)$ & 0.061 \\
\hline Uric acid (umol/L) & $266.62(206.55-323.25)$ & $258.93(204.40-318.27)$ & $296.00(232.00-349.35)$ & 0.114 \\
\hline Urea nitrogen $(\mathrm{mmol} / \mathrm{L})$ & $3.74(3.00-4.80)$ & $3.60(2.90-4.42)$ & $4.43(3.42-5.81)$ & 0.258 \\
\hline Glucose $(\mathrm{mmol} / \mathrm{L})$ & $6.30(5.44-7.81)$ & $6.24(5.41-7.69)$ & $6.51(5.57-8.95)$ & 0.815 \\
\hline Cholesterol & $3.90(3.41-4.59)$ & $3.90(3.33-4.51)$ & $4.02(3.55-4.76)$ & 0.133 \\
\hline Triglyceride & $1.23(0.92-1.96)$ & $1.22(0.88,1.89)$ & $1.32(0.96-2.38)$ & 0.209 \\
\hline HDL & $1.14(0.95-1.33)$ & $1.13(0.94-1.33)$ & $1.15(1.01-1.34)$ & 0.724 \\
\hline LDL & $2.15(1.77-2.68)$ & $2.17(1.74-2.67)$ & $2.12(1.82-2.78)$ & 0.267 \\
\hline \multicolumn{5}{|l|}{ Chest radiographs and CT finding } \\
\hline Unilateral pneumonia & $10(2.6)$ & $10(3.2)$ & $0(0)$ & 0.220 \\
\hline Bilateral pneumonia & $341(87.7)$ & $268(85.6)$ & $73(97.3)$ & $<0.001$ \\
\hline Normal & $16(4.1)$ & & $2(2.7)$ & 0.747 \\
\hline
\end{tabular}

Table 2. Laboratory findings of patients infected with COVID-19 on admission. CRP C-reactive protein; ESR erythrocyte sedimentation rate; $H D L$ high-density lipoprotein; $h s-C R P$ high sensitivity C-reactive protein; IQR interquartile range; $L D L$ low density lipoprotein.

(11.2 vs. 17.3\%, $p<0.001$ ), hypoproteinemia (4.8 vs. 17.3\%, $p<0.001)$ and ARDS (2.6 vs. 8.0\%, $p<0.001)$, while complications such as anemia, pneumonia, arrhythmia and leukopenia were insignificantly altered (Table 3 ).

HTN and cardiac injury. Next, we sought to investigate the association between blood pressure and cardiac injury. As seen in the Fig. 1, patients with HTN demonstrated a significant increase in the level of cardiac injury markers - CK, myoglobin, LDH, and NT-pro BNP, while change in CK-MB was subtle. Correlation analysis revealed that systolic blood pressure in patients with COVID-19 correlated significantly with the levels of CK $(\mathrm{R}=0.124, p=0.01)$, and $\mathrm{LDH}(\mathrm{R}=0.103, p=0.05)$; while diastolic blood pressure showed a trend to correlate with the level of myoglobin $(\mathrm{R}=0.113, p=0.06)$ and LDH $(\mathrm{R}=0.089, p=0.09)$ (Fig. 2). Further logistic regression analysis showed that HTN was associated with increased LDH $(p=0.001)$ and CK-MB $(p=0.05)$ in COVID- 19 patients after adjusting age, gender, and comorbidity of diabetes, coronary heart disease, chronic obstructive pulmonary disease, cerebrovascular disease, and chronic kidney disease (Table 4).

Previous studies have demonstrated that inflammatory cells such as macrophage and T cells in HTN infiltrate into the heart and results in cardiac and other end-organ damage ${ }^{20}$. A significant correlation between cardiac injury (levels of $\mathrm{CK}$, myoglobin, $\mathrm{LDH}$, and $\mathrm{CK}-\mathrm{MB}$ ) and inflammation (CRP, hs-CRP) has been observed in the present study (Fig. 3). We next compared cardiac injury markers in hypertensive-COVID-19 patients with and without CRP/hsCRP incensement and found that patients who presented an elevated inflammatory status also demonstrated a significant increase in LDH and a moderate increase in CK $(p=0.07)$ and CK-MB $(p=0.09)$ (Fig. 4). Further correlation analysis suggested a significant correlation between hs-CRP and systolic blood pressure in patients with COVID-19 (Fig. 5). Those results suggested that HTN was associated with increased cardiac injury, and inflammation might participate in hypertensive mediated cardiac injury. 


\begin{tabular}{|l|l|l|l|r|}
\hline Variables & Total $(\mathbf{n}=\mathbf{3 8 8})$ & Normotension $(\mathbf{n}=\mathbf{3 1 3})$ & Hypertension $(\mathbf{n}=\mathbf{7 5})$ & $\boldsymbol{p}$ value \\
\hline Time of hospitalization, mean (SD), d & $16.4 \pm 7.3$ & $15.9 \pm 7.1$ & $18.7 \pm 8.0$ & $<0.001$ \\
\hline $\begin{array}{l}\text { Time from symptom onset to hospital admission, mean } \\
\text { (SD) }\end{array}$ & $5.4 \pm 3.6$ & $5.3 \pm 3.6$ & $5.8 \pm 3.8$ & 0.399 \\
\hline Severity of illness (n, \%) & \multicolumn{5}{|l|}{} \\
\hline Severe & $56(11.4)$ & $38(12.1)$ & $57(76)$ & $<0.001$ \\
\hline Mild & $332(85.3)$ & $275(87.9)$ & $18(24)$ & \\
\hline Complications (n, \%) & \multicolumn{5}{|l|}{} & 0.931 \\
\hline Drug-induced hepatitis & $32(8.2)$ & $26(8.3)$ & $6(8.0)$ & $\leq 0.05$ \\
\hline Electrolyte disorder & $48(12.3)$ & $35(11.2)$ & $13(17.3)$ & $<0.001$ \\
\hline Hypoproteinemia & $28(7.2)$ & $15(4.8)$ & $13(17.3)$ & 0.560 \\
\hline Anemia & $20(5.1)$ & $15(4.8)$ & $5(6.7)$ & 0.401 \\
\hline Pneumonia & $22(5.7)$ & $16(5.1)$ & $6(8.0)$ & 0.998 \\
\hline Arrhythmia & $7(1.8)$ & $6(1.9)$ & $1(1.3)$ & 0.363 \\
\hline Leukopenia & $8(2.1)$ & $8(2.6)$ & $0(0)$ & $\leq 0.05$ \\
\hline Acute respiratory distress syndrome & $14(3.6)$ & $8(2.6)$ & $6(8.0)$ & \\
\hline
\end{tabular}

Table 3. Complications of patients infected with COVID-19.
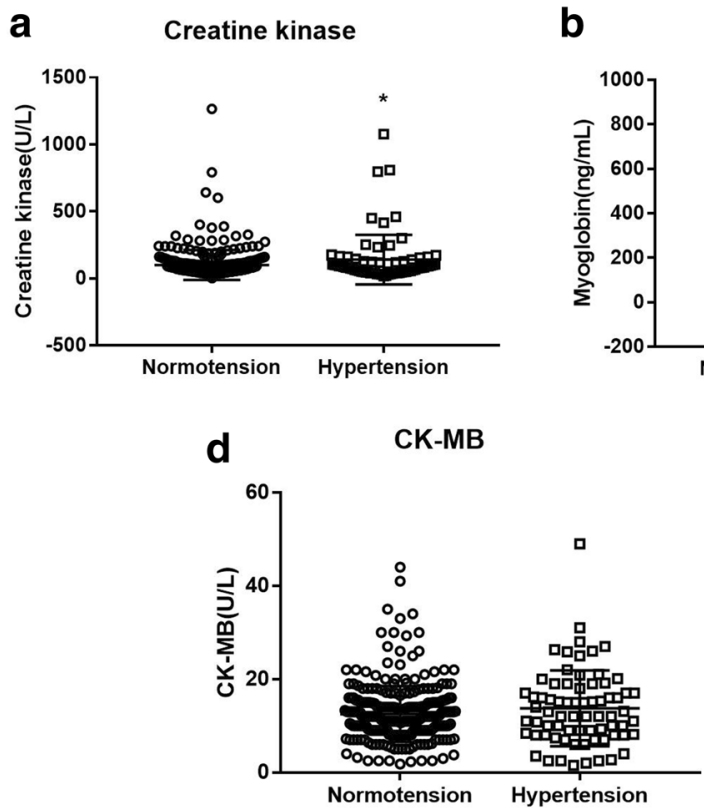

b

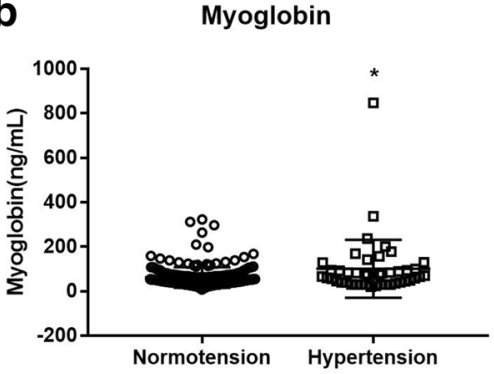

C

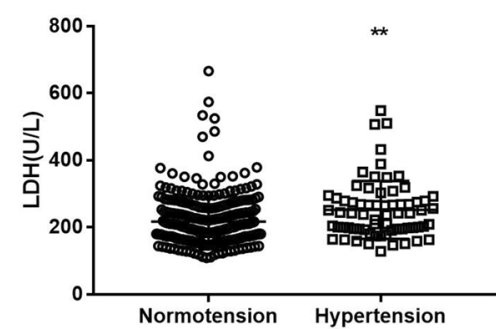

Figure 1. Cardiac injury markers in COVID-19 patients with and without hypertension. Caption: $C K$ creatine kinase; $C K-M B$ creatine kinase isoenzymes; $L D H$ lactate dehydrogenase; $N T$-proBNP N-terminal pronatriuretic peptide. Data was presented as mean $\pm \mathrm{SD}^{*}, p<0.05,{ }^{* *}, p<0.01,{ }^{* *}, p<0.001$ : COVID-19 patients with $v s$. without hypertension.

\section{Discussion}

To the best of our knowledge, this is the first report providing comprehensive information on epidemiological, demographic, clinical, laboratory and radiological characteristics of hospitalized COVID-19 patients in the context of HTN and ensuing myocardial damage. Our results specifically highlighted a significant association between HTN and cardiac injury in COVID-19 patients. In addition, our subgroup analysis demonstrated that inflammation could be a potential factor driving HTN induced cardiac injury in COVID-19.

The case-fatality rate for overall COVID-19 ranges from 0.0 to $28.9 \%$ across different geographical locations, while it rises up to $40 \%$ in patients admitted to intensive care unit ${ }^{21}$. Most importantly, cardiac injury has been reported as an independent risk factor of mortality in patients with COVID-19 ${ }^{12,22}$. A recent meta-analysis showed that $24.4 \%$ of the hospitalized COVID-19 patients developed cardiac injury and $72.6 \%$ of them did not survive $^{23}$. Studies also showed that hospitalized, COVID-19 patients with preexisting cardiovascular disease, such as HTN have been more susceptible to COVID-19 related cardiac injury ${ }^{23,24}$. Consistently, our study also demonstrated that HTN was the most common comorbidity (almost 19.3\%) observed in hospitalized COVID19 patients and also, these patients displayed an increase of cardiac injury markers, such as LDH, CK, and myoglobin. 

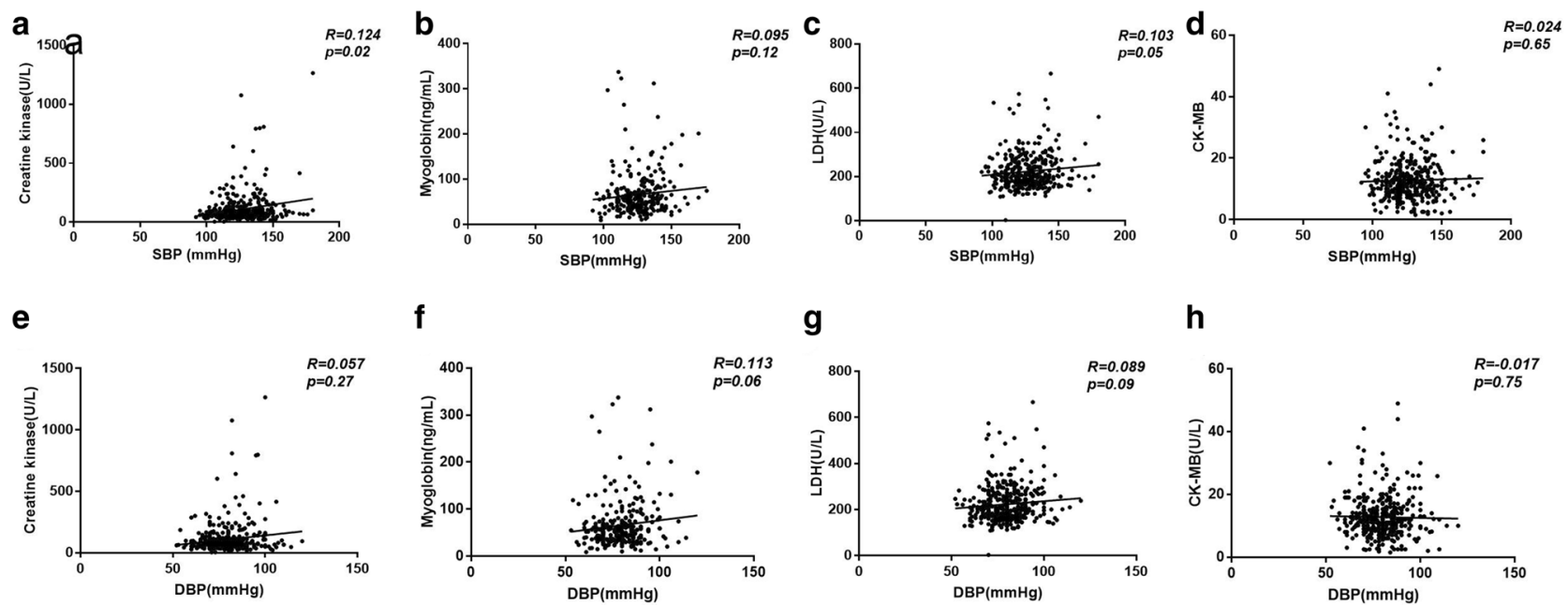

f

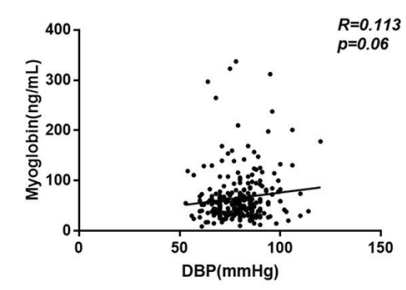

g

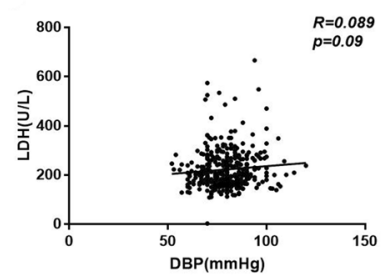

h

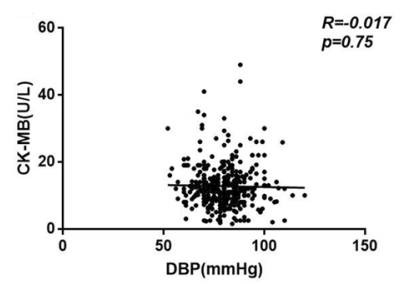

Figure 2. Correlation between blood pressure and cardiac injury markers in COVID-19. Caption: $C K$ creatine kinase; $C K-M B$ creatine kinase isoenzymes; $D B P$ diastolic blood pressure; $L D H$ lactate dehydrogenase; $S B P$ systolic blood pressure; NT-proBNP N-terminal pronatriuretic peptide.

\begin{tabular}{|c|c|c|c|c|}
\hline \multirow[b]{2}{*}{ Variable } & \multicolumn{2}{|l|}{ Crude } & \multicolumn{2}{|l|}{ Model I } \\
\hline & OR (95\%CIs) & $p$ value & OR (95\%CIs) & $p$ value \\
\hline \multicolumn{5}{|c|}{ Increased CK (NR:25-200U/L) } \\
\hline \multicolumn{5}{|l|}{ Blood pressure } \\
\hline Normotension & 1 (ref) & & 1 (ref) & \\
\hline Hypertension & $1.70(0.80,3.55)$ & 0.17 & $1.39(0.60,3.25)$ & 0.59 \\
\hline \multicolumn{5}{|c|}{ Increased Myoglobin (NR:0-85 ng/mL) } \\
\hline \multicolumn{5}{|c|}{ Blood pressure } \\
\hline Normotension & 1 (ref) & & 1 (ref) & \\
\hline Hypertension & $2.75(1.34,5.65)$ & 0.006 & $1.70(0.76,3.82)$ & 0.14 \\
\hline \multicolumn{5}{|c|}{ Increased CK-MB (NR:0-24U/L) } \\
\hline \multicolumn{5}{|c|}{ Blood pressure } \\
\hline Normotension & 1 (ref) & & 1 (ref) & \\
\hline Hypertension & $2.74(1.09,6.88)$ & 0.03 & $2.50(0.98,6.39)$ & 0.05 \\
\hline \multicolumn{5}{|c|}{ Increased LDH (NR:95-250U/L) } \\
\hline \multicolumn{5}{|l|}{ Blood pressure } \\
\hline Normotension & 1 (ref) & & 1 (ref) & \\
\hline Hypertension & $2.64(1.53,4.57)$ & 0.001 & $2.64(1.53,4.57)$ & 0.001 \\
\hline
\end{tabular}

Table 4. Relationship between hypertension against markers of cardiac injury. Caption: $C K$ creatine kinase; $C K-M B$ creatine kinase isoenzymes; $L D H$ lactate dehydrogenase; Crude model adjusted for none. Model I adjusted for age, gender, and comorbidity of Diabetes, Coronary heart disease, Chronic obstructive pulmonary disease, Cerebrovascular disease, and Chronic kidney disease.

As one of the most common cardiovascular comorbidities, HTN is a risk factor for increased intensive care unit admission ${ }^{14}$, utilization of mechanical ventilation ${ }^{25}$, and mortality ${ }^{26}$. A blood pressure control with a target of $<130 / 80 \mathrm{mmHg}$ during hospitalization is associated with fewer adverse clinical events in patients with COVID-1927. High blood pressure is one of the most critical risk factors for developing cardiovascular complications $^{28,29}$. In this study, we have demonstrated that systolic blood pressure in patients with COVID-19 correlated significantly with the levels of $\mathrm{CK}, \mathrm{LDH}$; and hypertension was associated with increased $\mathrm{LDH}$ and CK-MB in COVID- 19 patients after adjusting age, gender, and comorbidity of diabetes, coronary heart disease, chronic obstructive pulmonary disease, cerebrovascular disease, and chronic kidney disease. Thus, our study provided direct evidence in establishing a strong association between HTN and cardiac injury in COVID-19.

COVID-19 infection activates an excess production of inflammatory cytokines (interleukin- 6 and tumour necrosis factor- $\alpha$ ) - a cytokine storm which results in systemic inflammation, multi organ dysfunction and acutely, affecting the cardiovascular system ${ }^{30,31}$. An uncontrolled and dysfunctional immune response characterized by continuous activation and proliferation of lymphocytes and macrophages are the hallmark of this cytokine 
a
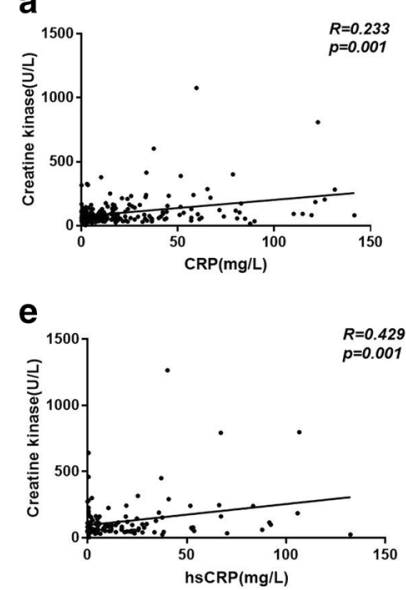

b

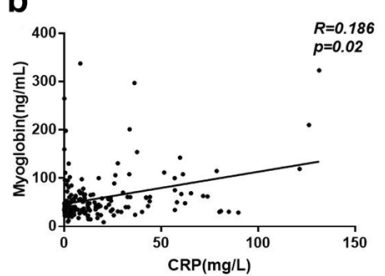

f

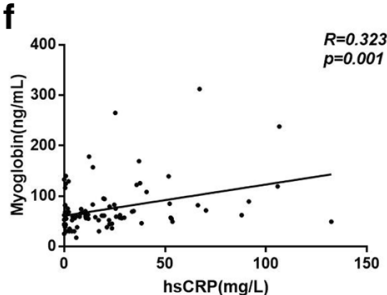

C

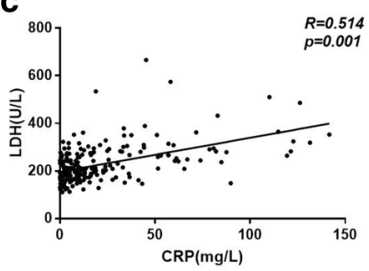

g

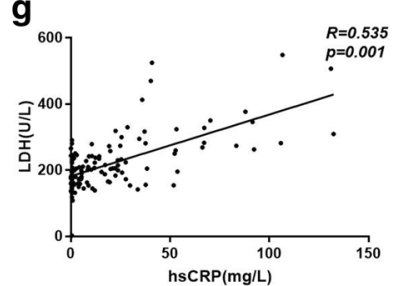

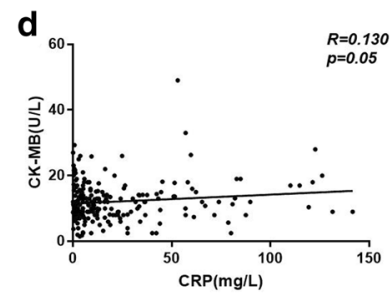

h

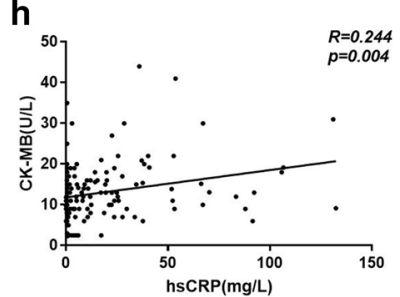

Figure 3. Correlation between CRP/hsCRP and cardiac injury markers in COVID-19. Caption: CK creatine kinase; $C K-M B$ creatine kinase isoenzymes; $C R P C$-reactive protein; $h s C R P$ high sensitive C-reactive protein; $L D H$ lactate dehydrogenase; NT-proBNP N-terminal pronatriuretic peptide.
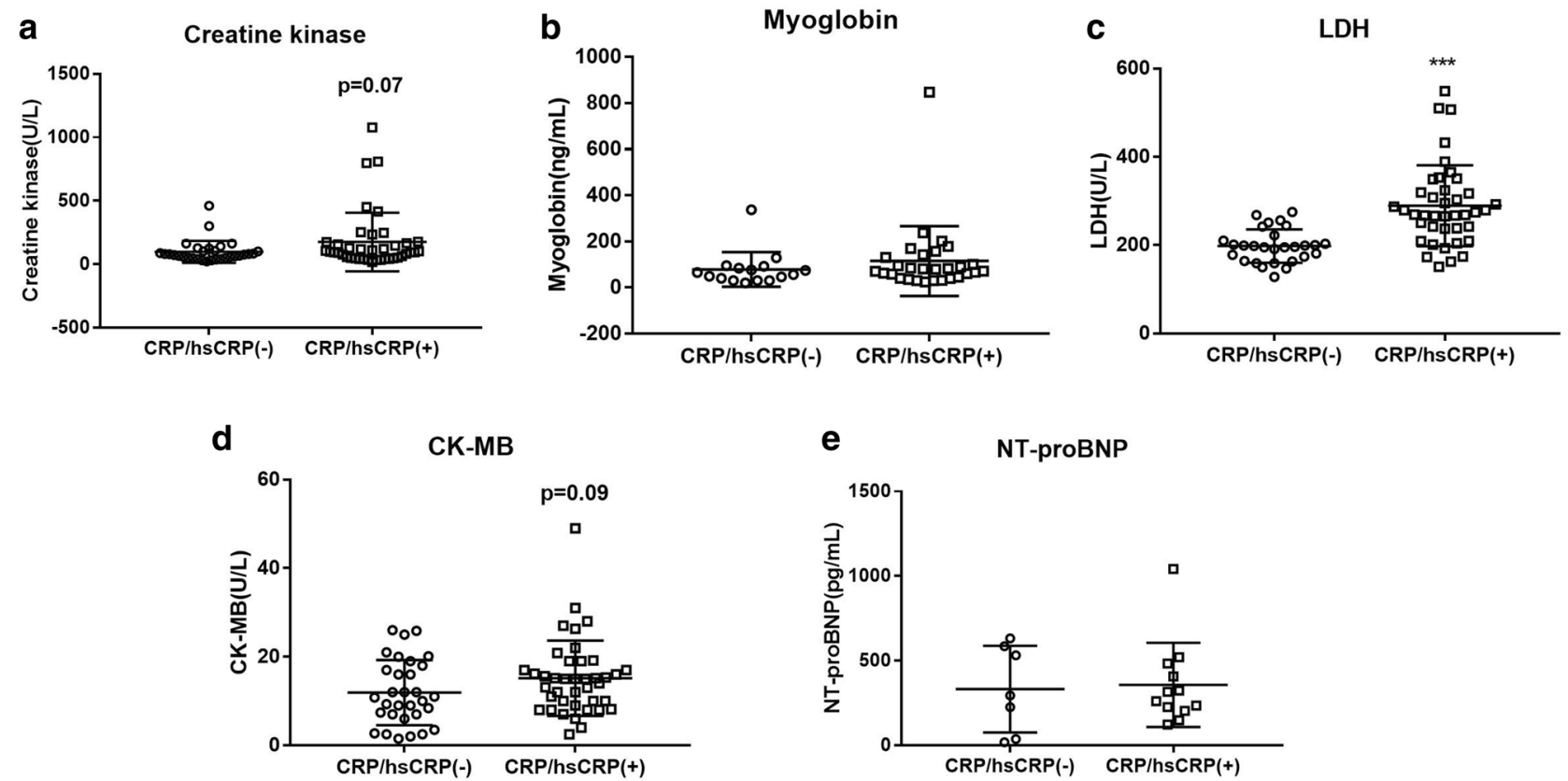

Figure 4. Cardiac injury markers in hypertensive COVID-19 patients with and without increased CRP/hsCRP. Caption: $C K-M B$ creatine kinase isoenzymes; $C R P$ C-reactive protein; $h s C R P$ high sensitive C-reactive protein; $L D H$ lactate dehydrogenase; NT-proBNP N-terminal pronatriuretic peptide. Data was presented as mean $\pm \mathrm{SD}$. ${ }^{* * *}, p<0.001$ : COVID-19 patients with $v s$. without hypertension.

storm syndrome ${ }^{32}$. In line with this, several autopsy reports have demonstrated an exaggerated infiltration of mononuclear inflammatory cells into the myocardia of COVID-19 patients, who presented higher viral load ${ }^{18,33,34}$.

In this study, we found inflammatory markers such as CRP, hs-CRP were significantly increased in hypertensive-COVID-19. Further, in the hypertensive group, COVID-19 patients with increased CRP or hs-CRP presented an increased level of cardia injury markers, LDH $(p<0.001)$, CK $(p=0.07)$ and CK-MB $(p=0.09)$. Incidentally, chronic inflammation associated with HTN synergizes cytokine storm and worsens host immune surveillance in SARS-CoV-2 infection ${ }^{35,36}$. It has been established that macrophages and $\mathrm{T}$ cells infiltrate into the heart in response to HTN, which ultimately results in end-organ damage ${ }^{20}$. Further, the deficiency of CD8 + cytotoxic T cells effectively protect against hypertension-induced cardiac damage in COVID-19 ${ }^{37,38}$. A significant linear correlation between hs-CRP and systolic blood pressure has been observed in the present study, suggesting HTN associated pro- inflammatory state might drives cardiac injury that worsens the prognosis of patients with COVID-19. 

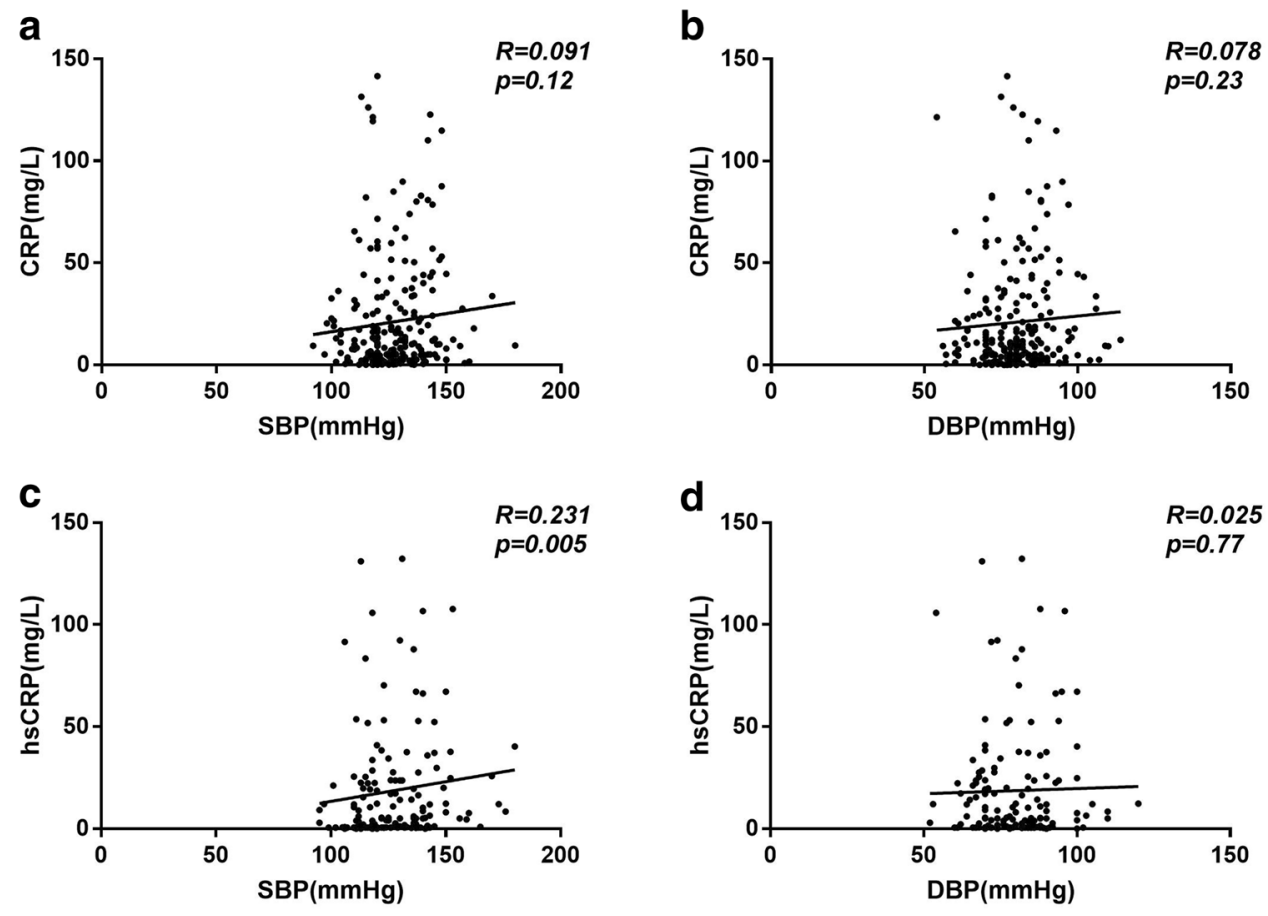

Figure 5. Correlation between blood pressure and CRP/hsCRP in COVID-19. Caption: CRP C-reactive protein; $h s C R P$ high sensitive C-reactive protein; $D B P$ diastolic blood pressure; $S B P$ systolic blood pressure.

We acknowledge that number of confirmed COVID-19 patients enrolled in this study is a limitation and further validation of our findings on a larger, multiple centered cohorts is warranted. Moreover, constrains such as space, time and the hospitals designated warded us off from utilizing conventional techniques such as echocardiography and electrocardiography.

\section{Conclusion}

Our investigation suggested that HTN is associated with a higher risk of cardiac injury and severe disease phenotype in COVID-19, effectively control blood pressure in HTN patients might improve the prognosis of COVID-19 patients. This study also suggested HTN associated pro- inflammatory state might drive cardiac injury that worsens the prognosis of patients with COVID-19, thus provided a cue in understanding the mechanism of cardiac injury in COVID-19.

\section{Data availability}

The datasets generated during and/or analyzed during the current study are available from the corresponding author on reasonable request.

Received: 7 June 2021; Accepted: 3 November 2021

Published online: 17 November 2021

\section{References}

1. Zhu, N. et al. A Novel coronavirus from patients with pneumonia in China, 2019. N. Engl. J. Med. 382, 727-733. https://doi.org/ 10.1056/NEJMoa2001017 (2020).

2. Shang, J. et al. Structural basis of receptor recognition by SARS-CoV-2. Nature 581, 221-224. https://doi.org/10.1038/s4158641020-42179-y (2020).

3. Coutard, B. et al. The spike glycoprotein of the new coronavirus 2019-nCoV contains a furin-like cleavage site absent in CoV of the same clade. Antiviral Res. 176, 104. https://doi.org/10.1016/j.antiviral.2020.104742 (2020).

4. Liu, P. P., Blet, A., Smyth, D. \& Li, H. The Science underlying COVID-19: Implications for the cardiovascular system. Circulation https://doi.org/10.1161/CIRCULATIONAHA.120.047549 (2020).

5. Plante, J. A. et al. Spike mutation D614G alters SARS-CoV-2 fitness. Nature 592, 116-121 (2021).

6. Li, B. et al. Prevalence and impact of cardiovascular metabolic diseases on COVID-19 in China. Clin. Res. Cardiol.: Off. J. German Cardiac Soc. 109, 531-538 (2020).

7. Wrapp, D. et al. Cryo-EM structure of the 2019-nCoV spike in the prefusion conformation. Science 367, 1260-1263. https://doi. org/10.1126/science.abb2507 (2020).

8. Santos, R. A., Ferreira, A. J., Verano-Braga, T. \& Bader, M. Angiotensin-converting enzyme 2, angiotensin- (1-7) and Mas: new players of the renin-angiotensin system. J. Endocrinol. 216, R1-R17. https://doi.org/10.1530/JOE-1512-0341.Print2013Feb (2013).

9. Imai, Y. et al. Angiotensin-converting enzyme 2 protects from severe acute lung failure. Nature 436, 112-116. https://doi.org/10. 1038/nature03712 (2005). 
10. Crackower, M. A. et al. Angiotensin-converting enzyme 2 is an essential regulator of heart function. Nature 417, 822-828. https:// doi.org/10.1038/nature00786 (2002).

11. Richardson, S. et al. Presenting characteristics, comorbidities, and outcomes among 5700 patients hospitalized with COVID-19 in the New York City Area. JAMA 323, 2052-2059. https://doi.org/10.1001/jama.2020.6775 (2020).

12. Shi, S. et al. Association of cardiac injury with mortality in hospitalized patients with COVID-19 in Wuhan, China. JAMA Cardiol. 5, 802-810 (2020).

13. Zhou, F. et al. Clinical course and risk factors for mortality of adult inpatients with COVID-19 in Wuhan, China: a retrospective cohort study. Lancet 395, 1054-1062. https://doi.org/10.1016/S0140-6736(1020)30566-30563 (2020).

14. Guan, W. J. et al. Clinical characteristics of coronavirus disease 2019 in China. N. Engl. J. Med. 382, 1708-1720. https://doi.org/ 10.1056/NEJMoa2002032 (2020).

15. Bolin, W., Ruobao, L., Zhong, L. \& Huang, Y. Does comorbidity increase the risk of patients with COVID-19: evidence from metaanalysis. Aging (Albany NY). 12, 6049-6057 (2020).

16. Fan, H. et al. Cardiac injuries in patients with coronavirus disease 2019: Not to be ignored. Int. J. Infect. Dis.: IJID: Off. Publ. Int. Soc. Infect. Dis. 96, 294-297 (2020).

17. Yao, X. H. et al. A pathological report of three COVID-19 cases by minimal invasive autopsies. Zhonghua bing li $x u e$ za $z h i=$ Chin. J. Pathol. 49, 411-417 (2020).

18. Xu, Z. et al. Pathological findings of COVID-19 associated with acute respiratory distress syndrome. Lancet Respir. Med. 8, 420-422 (2020).

19. (2020), W. H. O. Clinical management of severe acute respiratory infection when novel coronavirus (nCoV) infection is suspected: interim guidance. . 28 January 2020. World Health Organization. https://apps.who.int/iris/handle/10665/330893.

20. Frieler, R. A. \& Mortensen, R. M. Immune cell and other noncardiomyocyte regulation of cardiac hypertrophy and remodeling. Circulation 131, 1019-1030 (2015).

21. Wiersinga, W. J., Rhodes, A., Cheng, A. C., Peacock, S. J. \& Prescott, H. C. Pathophysiology, transmission, diagnosis, and treatment of coronavirus disease 2019 (COVID-19): a review. JAMA 324, 782-793 (2020).

22. Han, H. et al. Analysis of heart injury laboratory parameters in 273 COVID-19 patients in one hospital in Wuhan, China. J. Med. Virol. 92, 819-823 (2020).

23. Zou, F., Qian, Z., Wang, Y., Zhao, Y. \& Bai, J. Cardiac injury and COVID-19: a systematic review and meta-analysis. CJC open 2, 386-394 (2020).

24. Wei, J. F. et al. Acute myocardial injury is common in patients with COVID-19 and impairs their prognosis. Heart 106, 1154-1159 (2020).

25. El-Battrawy, I. et al. COVID-19 and the impact of arterial hypertension-An analysis of the international HOPE COVID-19 Registry (Italy-Spain-Germany). Eur. J. Clin. Investig. e13582 (2021).

26. Ferrari, R., Di Pasquale, G. \& Rapezzi, C. Commentary: what is the relationship between Covid-19 and cardiovascular disease?. Int. J. Cardiol. 310, 167-168 (2020).

27. Wang, T. et al. Predictors of fatal outcomes among hospitalized COVID-19 patients with pre-existing hypertension in China. Clin. Respir. J. 15, 915-924 (2021).

28. Stamler, J., Stamler, R. \& Neaton, J. D. Blood pressure, systolic and diastolic, and cardiovascular risks. US population data. Arch. Internal Med. 153, 598-615 (1993).

29. He, J. \& Whelton, P. K. Elevated systolic blood pressure and risk of cardiovascular and renal disease: overview of evidence from observational epidemiologic studies and randomized controlled trials. Am. Heart J. 138, 211-219 (1999).

30. Yang, C. \& Jin, Z. An acute respiratory infection runs into the most common noncommunicable epidemic-COVID-19 and cardiovascular diseases. JAMA Cardiol. 5, 743-744 (2020).

31. Aziz, M., Fatima, R. \& Assaly, R. Elevated interleukin-6 and severe COVID-19: a meta-analysis. J. Med. Virol. (2020).

32. Saverino, D. COVID-19: are T lymphocytes simply watching? Panminerva Medica (2020).

33. Liu, Y. et al. Clinical and biochemical indexes from 2019-nCoV infected patients linked to viral loads and lung injury. Sci. China Life Sci. 63, 364-374. https://doi.org/10.1007/s11427-11020-11643-11428 (2020).

34. Liu, K. et al. Clinical characteristics of novel coronavirus cases in tertiary hospitals in Hubei Province. Chin. Med. J. (Engl.) 133, 1025-1031. https://doi.org/10.1097/CM1029.0000000000000744 (2020).

35. Zheng, Z. et al. Risk factors of critical \& mortal COVID-19 cases: a systematic literature review and meta-analysis. J. Infect. 81, e16-e25. https://doi.org/10.1016/j.jinf.2020.1004.1021 (2020).

36. Azevedo, R. B. et al. Covid-19 and the cardiovascular system: a comprehensive review. J. Hum. Hypertens. 35, 4-11 (2020).

37. Zhang, C., Wang, F.-S., Silvestre, J.-S., Arenzana-Seisdedos, F. \& Tang, H. Is aberrant CD8+ T cell activation by hypertension associated with cardiac injury in severe cases of COVID-19?. Cell. Mol. Immunol. 17, 675-676 (2020).

38. Ma, F. et al. The requirement of CD8+ T cells to initiate and augment acute cardiac inflammatory response to high blood pressure. J. Immunol. (Baltimore, MD, 1950) 192, 3365-3373 (2014).

\section{Author contributions}

Project conceptualization and design; X.Z., H.C., Z.Y., data collection; D.L., Y.T., F.L., W.L.,; statistical analysis: L.Z., X.L., manuscript preparation; X.Z., A.R. All authors participated in the interpretation of data as well as critical revisions of the manuscript. H.C., Z.Y., and A.R. supervised the study.

\section{Funding}

Dr. Huiling Chen is supported by Natural Science Foundation of Hunan Province (2013SK3019, 2017SK2251) and National Natural Science Foundation of China (30771025); Dr. Xiaofang Zeng is supported by Fundamental Research Funds for the Central Universities of Central South University (1053320191678). Dr. Anandharajan Rathinasabapathy is funded by VUMC Faculty Research Scholars grant and R01HL095797-08. Dr. Zaixin Yu is supported by grants from National Natural Science Foundation of China $(81873416,82070055)$, and Key Research and development program of Hunan Province (2020SK2065). Dr. Xiangwei Liu is supported by grant from National Natural Science Foundation of China (81700279). Dr. Lihuang Zha is supported by grant from National Natural Science Foundation of China (8210012237). These funding have no role in study design, data collection, data analysis, data interpretation and manuscript preparation.

\section{Competing interests}

The authors declare no competing interests.

\section{Additional information}

Correspondence and requests for materials should be addressed to H.C. 
Reprints and permissions information is available at www.nature.com/reprints.

Publisher's note Springer Nature remains neutral with regard to jurisdictional claims in published maps and institutional affiliations.

(c) (i) Open Access This article is licensed under a Creative Commons Attribution 4.0 International License, which permits use, sharing, adaptation, distribution and reproduction in any medium or format, as long as you give appropriate credit to the original author(s) and the source, provide a link to the Creative Commons licence, and indicate if changes were made. The images or other third party material in this article are included in the article's Creative Commons licence, unless indicated otherwise in a credit line to the material. If material is not included in the article's Creative Commons licence and your intended use is not permitted by statutory regulation or exceeds the permitted use, you will need to obtain permission directly from the copyright holder. To view a copy of this licence, visit http://creativecommons.org/licenses/by/4.0/.

(C) The Author(s) 2021 